\title{
South African pilot study: End-to-end radiotherapy and medical physics dosimetry audits
}

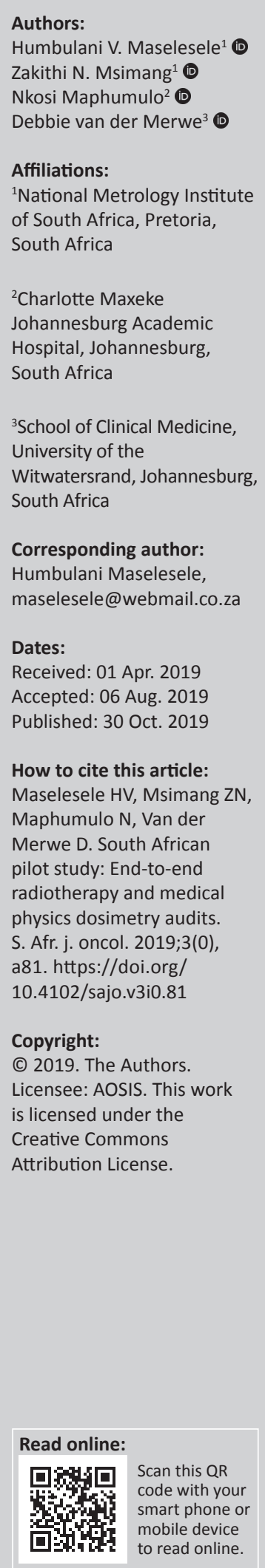

Background: The National Metrology Institute of South Africa (NMISA), as an institute responsible for disseminating traceability, has embarked on establishing a national dosimetry audit programme with assistance from the International Atomic Energy Agency (IAEA). Dosimetry audits in South Africa are carried out through the IAEA using mailed thermoluminescents. Onsite dosimetry is not performed in South Africa but the IAEA has provided a dosimetry kit to NMISA to facilitate the audits. The kit consists of a Farmer type PTW 30012 ionisation chamber for photon beams and an Advanced Markus PTW 34045 chamber for electron beams with a water phantom and a Perspex plate phantom. End-to-end audits are also not performed or, if performed, they are not monitored officially in South Africa; however, the IAEA also provided a CIRS IMRT thorax phantom which can be used nationally.

Aim: To test the establishment of radiotherapy and medical physics dosimetry audits at pilot centres in South Africa.

Setting: NIMISA was tasked by IAEA to establish radiotherapy and medical physics dosimetry audits. A calibration kit was supplied by the IAEA for dosimetry measurement and a CIRS IMRT thorax phantom was also supplied by the IAEA for end-to-end measurements. Five hospitals were identified by the steering committee as pilot centres to test the establishment of the dosimetry audits.

Methods: The audit measurements for reference beams were carried out using a Farmer type PTW 30012 ionisation chamber for photon beams and an Advanced Markus PTW 34045 chamber for electron beams with a water phantom and a Perspex plate phantom, respectively. End-to-end audits, which involved computed tomography (CT) scanning, treatment planning and treatment delivery with dose measurements, were carried out using a CIRS IMRT thorax phantom. The planning staff or the medical physicists were requested to image the phantom and create a treatment plan according to a procedure provided by the researcher on their institutional treatment planning system. On completion, the approved plan was delivered at the treatment unit by setting up the phantom and measuring the doses with an ionisation chamber at different specified reference points.

Results: For photon beam reference dosimetry, variations between $0.5 \%$ and $2.48 \%$ were observed, and for electron beams, variations of up to $8.0 \%$ were observed. For end-to-end audits, variations of up to $7.1 \%$ were observed between the calculated and measured doses.

Conclusion: The pilot audits provided a platform to formulate and validate the audit methodologies required for the establishment of a national end-to-end dosimetry audit programme.

Keywords: radiotherapy; medical physics; dosimetry; audits; pilot study.

\section{Introduction}

Radiotherapy has an important role in the medical field for the treatment of cancer. It is one of the applications in which humans are deliberately subjected to very high radiation doses. Radiation dosimetry plays a vital role in ensuring accurate dose delivery to the target area, achieving the required level of accuracy and maintaining consistency in dose delivery. ${ }^{1}$ In radiotherapy, the outcome of treatment greatly depends on the accuracy of dose delivery to the cancer site. This in turn depends on target volume delineation, radiation dosimetry, treatment planning and patient positioning as well as treatment delivery. ${ }^{2}$

The treatment planning process used in radiotherapy is complex and involves multiple steps and special dose calculation algorithms like pencil beam, convolution, superposition and so on. 
Specific quality assurance (QA) tests for treatment planning systems (TPSs) provide the framework and guidance to allow radiation oncology physicists to design comprehensive and practical treatment planning QA programmes for the clinics. ${ }^{3}$ It is essential to also audit clinical processes such as contouring where the body outline, targeted tumour volumes and critical structures are delineated. The TPS is used to predict the dose distribution from the beam arrangement on patient computed tomography (CT) scans. The dose calculation accuracy can also be verified using anthropomorphic phantoms. ${ }^{4,5}$ According to the International Commission on Radiological Protection (ICRP), ${ }^{6}$ severe toxicities or even fatal consequences can emerge from errors arising from incorrect radiation beam calibrations or a lack of independent verification of the calculated treatment times and monitor units (MU) for treatment delivery.

Radiation dosimetry deals with methods of quantitative determination of the energy deposited in a medium by directly or indirectly ionising radiations. Radiation measurements can be carried out using films, ionisation chambers, diodes, thermoluminescent dosimeters and other commercially available dosimeters. ${ }^{7,8}$

Various dosimetry quantities and methodologies have been developed to facilitate dose calculation in radiotherapy. Basic dose distribution data are usually measured in a water phantom, which closely approximates the radiation absorption and scattering properties of muscle and other soft tissues. Other materials like Perspex and solid water phantom plates can also be used. ${ }^{9,10}$ In South Africa, hospitals only participate in the International Atomic Energy Agency (IAEA) or Imaging and Radiation Oncology Core (IROC) reference beam dosimetry audits. A need to develop our own in-house dosimetry audits was established with the aim of expanding from reference beams to end-to-end audits to assist hospitals in identifying and reducing these errors.

Dosimetry audits play an important role in verifying the treatment chain in radiotherapy, thus ensuring safety and quality during cancer treatment delivery. Because of an increase in the number of radiotherapy treatment machines and the introduction of more advanced radiotherapy techniques, it is important to perform dosimetry audits to ensure the quality of dose delivery. ${ }^{11}$ Radiation dosimetry audit measurements can involve various approaches depending on the availability of radiotherapy equipment in the hospitals. Photon dosimetry audits for reference and non-reference conditions in conventional fields can be carried out using films, diodes and commercially available Farmer ionisation chambers, which measure absorbed dose in the specified medium. ${ }^{12}$

The International Atomic Energy Agency/World Health Organisation's (IAEA/WHO) thermoluminescent (TLD) dosimetry postal programme for external audits of the calibration of high-energy photon beams used in radiotherapy has been in operation since $1969 .{ }^{13}$ The IAEA has several postal dose audit services operating worldwide including the
European Society for Therapeutic Radiology and Oncology (ESTRO), EQUAL operating in the European Union (EU) and the Radiological Physics Center (RPC) in North America. The IAEA has developed various types and levels of external audits for radiotherapy dosimetry, either based on onsite review visits or using mailed dosimetry systems. They have analysed the combined relative standard uncertainty on dose determined from TLD measurements estimated to be $1.2 \%$ for irradiations with Co- 60 X-rays and 1.6\% for irradiations with high-energy $\mathrm{X}$-rays. ${ }^{14,15}$ Various groups including IAEA dosimetry laboratory, IROC in Houston, the Catalan Society of Medical Physicists and the Medical Physics group in the United Kingdom radiotherapy departments have developed a range of procedures used for external dosimetry audits. These include international QA networks for radiotherapy dosimetry, multicentre validation of intensity modulated radiotherapy (IMRT), pre-treatment verification and a national dosimetry audit on IMRT. ${ }^{16,17}$

Radiation dosimetry audits for reference beams serve as a tool to verify dose output of teletherapy units.

Other centres have developed in-house methods for performing different types of audits based on the availability of equipment. ${ }^{18}$ In South Africa, reference beam output is determined according to the IAEA, Technical Report Series number 398 (IAEA TRS 398) Protocol for the Absorbed Dose Determination in External Beam Radiotherapy. Photon beam outputs are determined at a reference depth of $10 \mathrm{~cm}$ in a water phantom, and electron beams are also calibrated at a specified reference depth given by $Z_{\text {ref }}=$ $0.6 R_{50}+0.1$ g.cm ${ }^{-2}$ in water. The $50 \%$ beam quality $\left(R_{50}\right)$ specifier is obtained from depth ionisation scans provided by the audited hospital. ${ }^{19}$

The IAEA is mostly responsible for Europe, Africa, Asia and even other continents. However, institutions, such as the IROC in Houston, are responsible for audits in America; they have also conducted both remote and onsite audits. Their 45-year history has accumulated extensive measurement data from several thousand photon beams which have been grouped into 96 combinations of manufacturer model and beam energy.

Their presented results have shown that most audits were within the $\pm 5 \%$ range with a few outside this range. ${ }^{20,21}$ The National Metrology Institute of South Africa (NMISA), as the institute that provides measurement traceability, embarked on establishing a national dosimetry audit programme for radiotherapy and medical physics with assistance from the IAEA. Five centres with different types of linear accelerators (linacs) were identified to participate in the pilot study for the establishment of the dosimetry audit programme.

\section{Design}

The initial steps for the pilot study aimed at conducting a survey of the equipment available in the selected centres, including the beam data that are used for defining the 
specified radiation beam quality factors. The questionnaire, a consent request for authorisation to perform audits and a form to collect radiation beam data characteristics such as the percentage depth dose (PDD) or tissue phantom ratio $\left(\mathrm{TPR}_{20,10}\right.$ ) of the dose at depth $20 \mathrm{~cm}$ to depth $10 \mathrm{~cm}$ for photon beams, and the $50 \%$ beam quality $\left(R_{50}\right)$ specifier for electron beams, were sent to each of the pilot centres. The scope of the pilot study was to perform the following measurements:

- Reference dose output measurements in water according to the IAEA TRS 398 code of practice, for both photon and electron beams on the linear accelerators. ${ }^{19}$

- Radiation dose output measurements for nonreference conditions including measurements of field size dependence and measurements in the presence of a wedge..$^{22,23}$

- An end-to-end radiotherapy audit using an anthropomorphic phantom following the IAEATECDOC-1583 guidelines. ${ }^{24}$

After receiving the completed questionnaire and authority to perform the audit measurements from the hospital, an appointment was secured with the centre. The audit visit was scheduled for at least 2 days.

The expectation was that reference beam audits will be performed in the presence of the local medical physicist, while end-to-end dosimetry audits will be performed in the presence of both the local medical physicist and radiation therapists. This pilot audit study did not include auditing of clinical radiation oncology activities. The five participating hospitals were labelled as 1 (with 1 A representing linac 1 and 1B representing linac 2), 2 (one linac), 3 (one linac) and 4 (4A linac 1 and 4B linac 2) and 5. This was to ensure the anonymity of the participating hospitals.

\section{Materials and methods Reference condition measurements}

A dosimetry system consisting of a PTW 30012 Farmer type cylindrical ionisation chamber, PTW T1002 unidos electrometer, Lutron PHB-131 barometer, Yuwese DWL series thermometer and a locally produced $30 \mathrm{~cm} \times 30 \mathrm{~cm} \times 30 \mathrm{~cm}$ water phantom made of $1-\mathrm{cm}$ thick Perspex walls was used. For electron beam measurements, an Advanced Markus chamber PTW 34045 was used in water with adjustable height. An alternative method was tested using equivalent phantom made up of $30 \mathrm{~cm} \times 30 \mathrm{~cm}$ Perspex plates of thicknesses $0.1 \mathrm{~cm}, 0.2 \mathrm{~cm}, 0.5 \mathrm{~cm}$ and $1 \mathrm{~cm}$. The ionisation chamber was calibrated at the national SSDL for treacebility. The method of using a Perspex plate phantom for the electron beams was adopted in order to accurately scale the measurement depth. As a general principle, equipment was left to stabilise to ambient conditions for about an hour before any measurements commenced. Linear accelerators audited during this pilot study included two Varian DMX 2100C, two Elekta Precise and four Siemens Primus units from the five different hospitals.

\section{Photon beam setup}

The water phantom was placed on the couch top and set up for vertical irradiation with the linear accelerator gantry and collimator positioned at 0 degrees. For hospitals using source axis distance (SAD) setup, the reference point of the ionisation chamber labelled $Z_{\text {ref }}$ was positioned at $10 \mathrm{~cm}$ depth, and the source surface distance (SSD) was set at $90 \mathrm{~cm}$ (Figure 1). ${ }^{1}$ For SSD setup, the reference point was set at $10 \mathrm{~cm}$ depth and the SSD was set at $100 \mathrm{~cm}$.

The set field size was $10 \mathrm{~cm} \times 10 \mathrm{~cm}$ at isocenter for SAD technique and at the water surface for SSD technique. A calibrated thermometer and a barometer were used to measure the temperature and pressure measurements, respectively. Using the ionisation chamber connected to the electrometer, charge measurements were taken with a beam on time of $100 \mathrm{MU}$ on the linear accelerator. Equation 1 was used to calculate the absorbed dose at a depth of maximum dose for the SSD setup and Equation 2 was used for the SAD setup

$D_{w, Q}\left(z_{\max }\right)=\frac{M_{1} \cdot k_{T P} \cdot k_{\mathrm{elec}} \cdot k_{\mathrm{pol}} \cdot k_{s} \cdot N_{D, w, Q_{0}} \cdot k_{Q, Q_{0}}}{P d d_{\mathrm{Zref}}}$ [Eqn 1]

$D_{w, Q}\left(z_{\max }\right)=\frac{M_{1} \cdot k_{T P} \cdot k_{\text {elec }} \cdot k_{\mathrm{pol}} \cdot k_{s} \cdot N_{D, w, Q_{0}} \cdot k_{Q, Q_{0}}}{T M R_{\text {Zref }}}$

[Eqn 2]

where $Z_{\max }$ is the depth of maximum dose. $Z_{\text {ref }}$ is the reference depth. $M_{1}$ is the charge reading. $K_{\text {elec }}$ is the electrometer calibration factor. $K_{\mathrm{pol}}$ is the polarity correction. $K_{\mathrm{s}}$ is the recombination correction. $N_{D, W, Q o}$ is the absorbed dose to water calibration factor for a dosimeter at reference beam quality $Q_{0}$.

$k_{\mathrm{Q}, \mathrm{QO}}$ is the beam quality correction factor that accounts for the difference between the reference beam quality $Q_{0}$ and the actual quality $Q$ being used. $k_{\mathrm{TP}}$ is the temperature and pressure correction factor calculated using Equation 3:

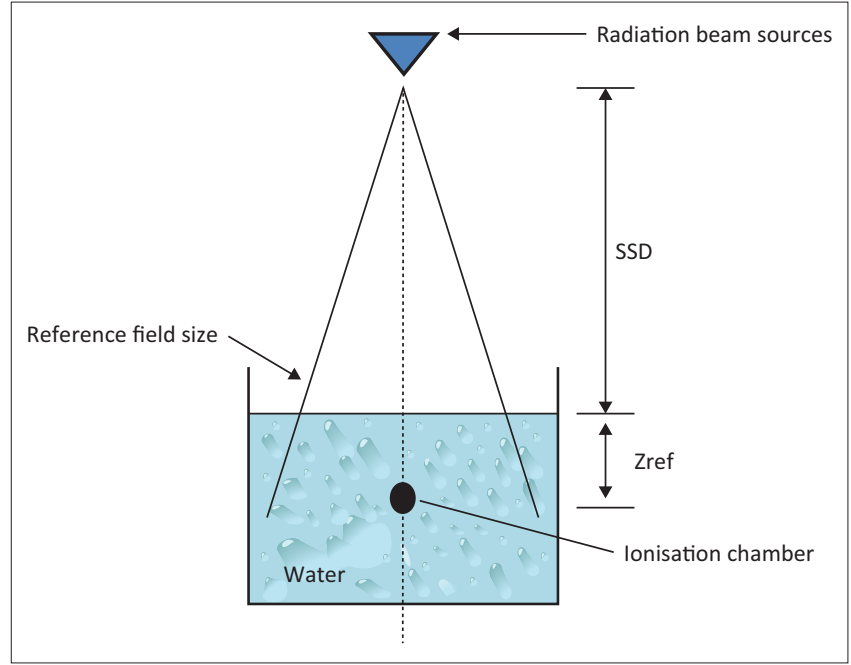

Source: Khan FM. The physics of radiation therapy. 5th ed. Philadelphia, PA: Lippincott William and Wilkins; Wolters Kluwer Health; 2009

SSD, source surface distance.

FIGURE 1: Geometric illustration of beam measurement setup. 
$k_{T P}=\frac{273.2+T}{273.2+T_{0}} \times \frac{P_{0}}{P}$

[Eqn 3]

$T_{o}$ and $P_{o}$ are standard temperature and standard pressure, respectively. $P d d_{\text {Zref }}$ is the PDD at reference depth. $T M R_{\text {Zref }}$ is the tissue maximum ratio at reference depth.

\section{Electron beam setup}

Electron measurement was carried out at calculated $Z_{\text {ref }}$ in water. The $R_{50}$ for each energy was determined from the PDD data provided by the hospital. The output of the beam for each selected energy was computed following the IAEA TRS $398 .^{19}$

An alternative cross-calibration method was used following the conversion mentioned in the AAPM TG 21 where for each energy, the $Z_{\text {ref }}$ was determined using the scaling factor of 0.88 for Perspex (Figure 2). ${ }^{1}$ This method was carried out after the dose output was measured in water as a means to cross calibrate the beam output in cases where the hospital does not have a water phantom of adjustable vertical movement. ${ }^{25,27}$

The reference depth in Perspex, $Z_{\text {ref, }, p^{\prime}}$ was therefore determined from the reference depth in water, $Z_{\text {ref, } w^{\prime}}$ using the scaling factor. $^{24}$

$Z_{\text {ref }, p}=Z_{\text {ref, } w} x S F(0.88)$

[Eqn 4]

The SSD was recalculated to compensate for the scaling factor and given as SSD1 using Equation 5.

$S S D 1=S D D+\left(Z_{\text {ref, } w}-Z_{\text {ref }, p}\right)$

In the final setup for the dosimetry audit measurement for the electron beams, the ionisation chamber was placed at $Z_{\text {ref, },}$ in the Perspex, and the SSD was set at the calculated value of SSD1. Charge measurements were taken using the ionisation chamber and the electrometer for a beam on time of $100 \mathrm{MU}$. All corrections as in Equations 1 and 2 above were applied following IAEA TRS 398 Protocol to determine the absorbed

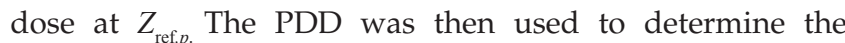
absorbed dose at $d_{\max }$ for electron beams. The results of this

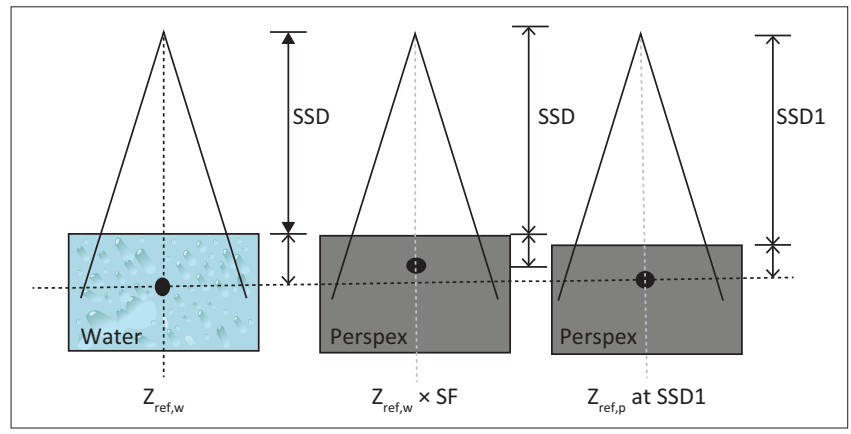

Source: Khan FM. The physics of radiation therapy. 5th ed. Philadelphia, PA: Lippincot William and Wilkins; Wolters Kluwer Health; 2009

SSD, source surface distance.

FIGURE 2: Geometric illustrations using scaling factor from water to Perspex measurements. method were within $1.0 \%$, and it was recommended that in case there is no phantom with vertical adjustment, this method can be considered if validated.

\section{Non-reference condition audit measurements}

Measurements for field size dependence (or output factors) were carried by taking radiation beam output measurements for different field sizes. Output factor measurements were carried out for field sizes $5 \mathrm{~cm}^{2}, 6 \mathrm{~cm}^{2}, 8 \mathrm{~cm}^{2}, 10 \mathrm{~cm}^{2}, 12 \mathrm{~cm}^{2}$, $15 \mathrm{~cm}^{2}$ and $20 \mathrm{~cm}^{2}$ for photon energies at $10 \mathrm{~cm}$ depth and $6 \mathrm{~cm}^{2}, 8 \mathrm{~cm}^{2}, 10 \mathrm{~cm}^{2}$ and $15 \mathrm{~cm}^{2}$ applicators for electron beams at specified $Z_{\text {ref }}$ for each applicator. The output factor was calculated using Equation 6.

Output Factor $=\frac{M_{f s}}{M_{\text {ref }, f s}}$

[Eqn 6]

where $M_{f s}$ is the reading for the selected field size and $M_{\text {ref } f, s}$ is the reading for the reference field size obtained in the $10 \mathrm{~cm} \times$ $10 \mathrm{~cm}$ field.

Wedge transmission output factors were obtained for photon beams by measuring output for a reference field size of $10 \mathrm{~cm} \times 10 \mathrm{~cm}$ with and without the wedge in place at $10 \mathrm{~cm}$ depth in water. The measurements were carried out at $90^{\circ}$ and $270^{\circ}$ with ionisation chamber position longitudinal to the wedge. The wedge transmission factor was then calculated using Equation 7.

Wedge Factor $=\frac{M_{\text {wedge }}}{M_{\text {ref }}}$

where $M_{\text {wedge }}$ is the reading with wedge, and $M_{\text {ref }}$ is the reading at reference condition, $10 \mathrm{~cm} \times 10 \mathrm{~cm}$ field size without the wedge. The results were compared with data provided by the hospital from TPS or commissioning measurement.

\section{End-to-end audit measurements}

In radiotherapy, different models of TPSs are used. In this pilot study, the TPSs audited included Eclipse, Oncentra, $\mathrm{XIO}$ and Monaco. In this audit procedure, the CIRS IMRT thorax phantom (Figure 3) ${ }^{24}$ was set up at the radiotherapy department's CT scanner using the three-point setup markers on the phantom. ${ }^{28}$ The phantom was scanned, and CT data transferred to the TPS for positioning verification, anatomical verifications and treatment planning preparations.

A three-field plan was prepared according to the procedure given on the IAEA-TECDOC-1583 guidelines, and the reference points were identified on a specified axial slice as shown in Figure 3(b). ${ }^{24,28}$

\section{Treatment plan parameters}

- Create a $15 \mathrm{~cm} \times 15 \mathrm{~cm}$ field at isocenter.

- Set gantry angle to $0^{\circ}$ and collimator rotation to $0^{\circ}$.

- Label the field as ANT.

- Create a second $15 \mathrm{~cm} \times 15 \mathrm{~cm}$ field at the same isocenter. 

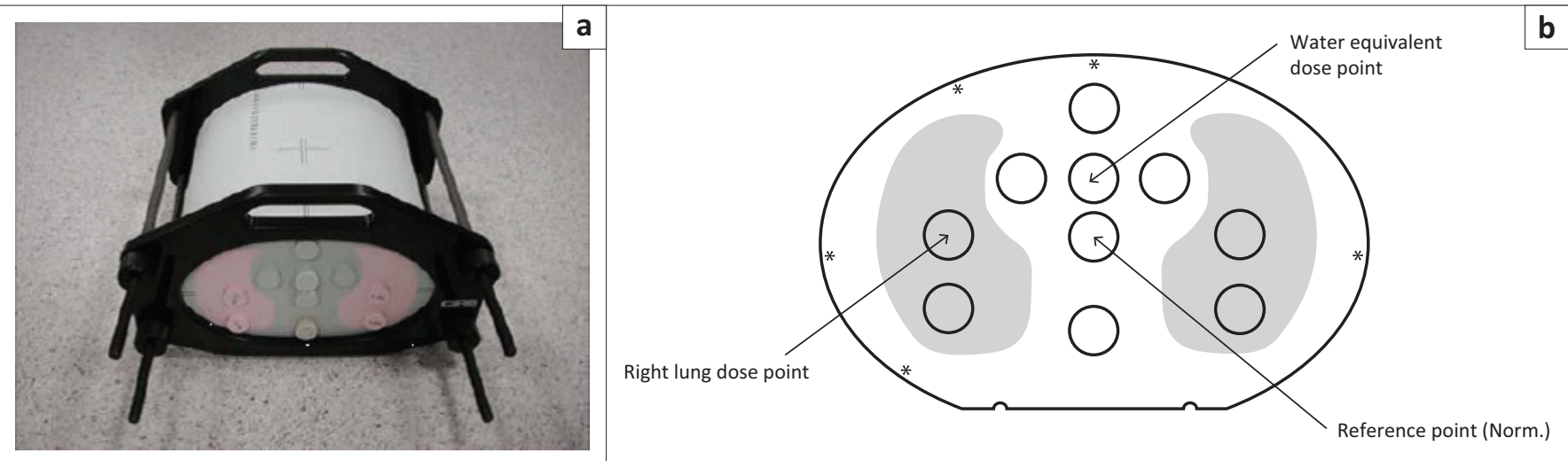

Source: Brunckhorst E, Gershkevitsh E, Ibbott G, et al. IAEA-TECDOC-1583. Commissioning of radiotherapy treatment planning systems: Testing for typical external beam treatment techniques. Vienna: International Atomic Energy Agency; 2008

FIGURE 3: (a) Thorax phantom (CIRS Model 002LFC) and (b) the reference points used during the dosimetry audit measurements.

- Set gantry to $110^{\circ}$ and collimator to $0^{\circ}$.

- Label the field as LPO.

- Create a third $15 \mathrm{~cm} \times 15 \mathrm{~cm}^{2}$ field at the same isocenter.

- Label the field as RPO.

- Set gantry to $250^{\circ}$ and collimator to $0^{\circ}$.

- Set prescription to $1 \mathrm{~Gy}$ at isocenter with equal field weighting.

- Calculate TPS MU/time needed to deliver 1 Gy to the reference point.

- Report the computed dose at reference point (Iso).

- Compute the dose at the 'Water equivalent dose point', 'Reference point norm' and 'Right lung dose point' as in Figure 4.

- Report the computed dose at created dose points.

\section{Treatment delivery}

- Prepare the plan for treatment and set up the phantom on the couch of the treatment machine with same orientation as in CT (head first supine) towards gantry.

- Set the gantry and collimator as in treatment planning report.

- Align the phantom with lasers intersection at the centre of the phantom as in CT scan.

- Set the isocenter to the $\mathrm{X}, \mathrm{Y}$ and $\mathrm{Z}$ coordinates obtained in planning.

- Verify the SSD.

- Ensure that the light field is not passing through the couch on any of the fields.

- Insert the Farmer ionisation chamber at the reference position using the correct insert holder - light blue (Make sure the calibration equipment is allowed enough time to settle to room condition).

- Deliver the treatment plan to the phantom and measure the dose to reference point from each field contribution.

- Record the value of the measured doses. Repeat irradiation at least three times for statistical evaluation.

- Insert the Farmer ionisation chamber at the 'Water equivalent dose point' position using the correct insert holder (light blue).

- Deliver the treatment plan to the phantom and measure the dose to reference point from each field contribution.

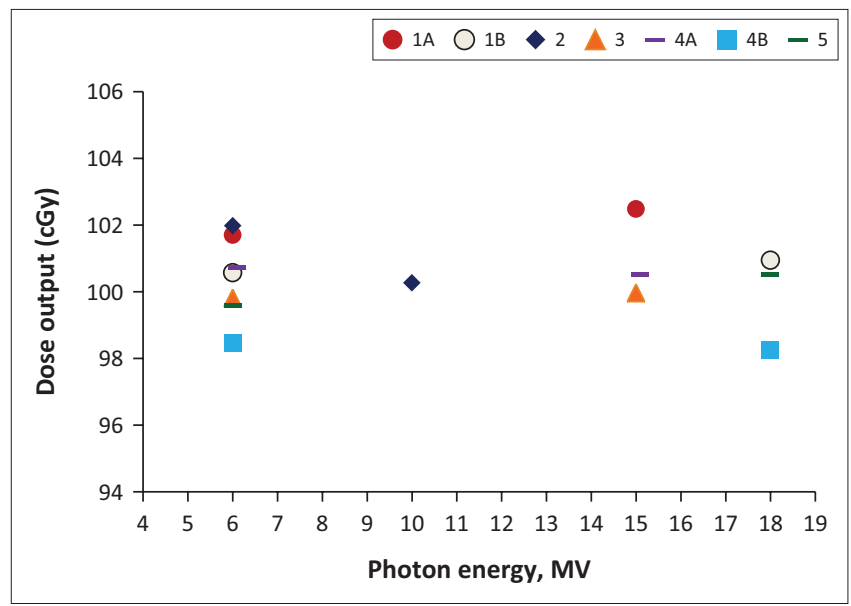

FIGURE 4: Photon beam output, TRS 398, for five hospitals (1A, 1B, 2, 3, 4A, $4 \mathrm{~B}$ and 5$)$.

- Register the value of the measured doses. Repeat irradiation at least three times.

- Insert the Farmer ionisation chamber at the 'Right lung dose point' position using the correct insert holder (pink).

- Deliver the treatment plan to the phantom and measure the dose to reference point from each field contribution.

- Register the value of the measured doses. Repeat irradiation at least three times.

\section{Ethical considerations}

No patient data were used in this study. A waiver from ethics clearance was received for this study from the Human Research Ethics Committee of the University of the Witwatersrand (W-CJ-171916-2). Consent letters providing approval to access radiotherapy equipment at each hospital were received.

\section{Results and discussions Initial beam data collection}

The collected beam data, which is PDD and $\mathrm{TPR}_{20,10}$ from different hospitals before onsite visit, were compared for the same photon energies. When comparing these beam data amongst the hospitals, the variation of PDD and $\mathrm{TPR}_{20,10}$ 
supplied values for the photon beams was found to be within $0.7 \%$ and $4.5 \%$, respectively.

\section{Reference beam data audits}

The results for the reference dose output as measured by the auditor for the photon and electron beams (Figures 4 and 5 , respectively) for the five pilot hospitals (1 [1A and 1B], 2, 3, 4 [4A and $4 \mathrm{~B}$ ] and 5) are presented as a function of the nominal beam energy. Dose output measurements were only taken once during the onsite visit, and only hospitals with disputed results took other measurements with their own equipment.

Under photon beam reference conditions, the minimum difference between the expected and measured beam outputs was in the range between $0.3 \%$ and $2.5 \%$.

The agreement in the electron reference beam output ranged from $0.15 \%$ to $8.0 \%$. No trend was observed with energy except on hospital 2, which showed better agreement at midrange energies. The variation could be attributed to the frequency of use; however, the main contributing factor was found to be coming from the different calibration setup and the use of a cylindrical chamber versus parallel-plate chamber. Some centres did not use a parallel-plate chamber, and this may have contributed to the increased error margin, especially at low energies as a cylindrical ionisation chamber is not recommended for electron energies $<10 \mathrm{MeV}$. Because of lack of calibration facilities at the local calibration laboratories, most centres with a parallel-plate chamber did not have updated calibration records, and this also contributed to increase in error margin.

\section{Non-reference conditions}

For this audit test, the measured data for the output factors for both low-energy and high-energy photon beams were compared with the data received from the pilot hospital (Figure 6) for each corresponding linear accelerator.

The measured output factors were compared with the data collected for the minimum field size of $5 \mathrm{~cm}^{2}$ to the maximum field size of $20 \mathrm{~cm}^{2}$. The agreement varied from $-4.7 \%$ to just below $2.2 \%$.

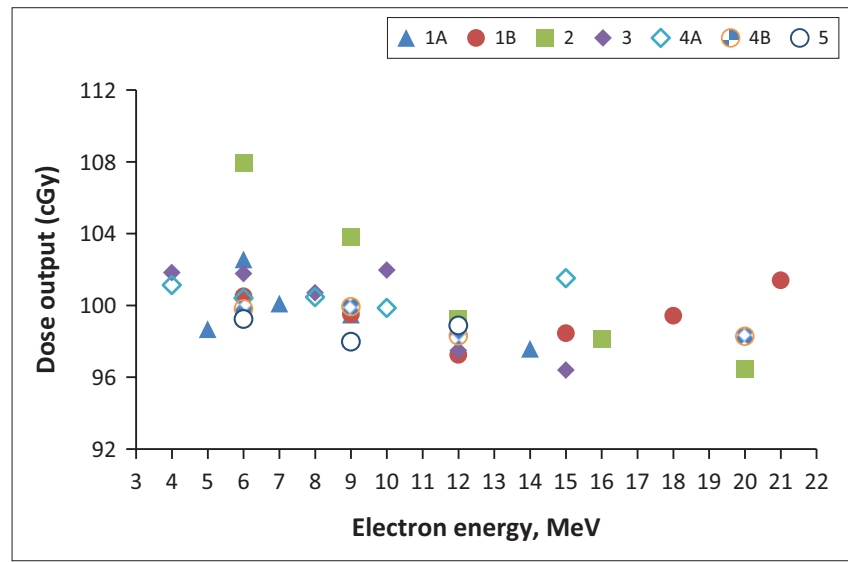

FIGURE 5: Electron beam output for five hospitals (1A, 1B, 2, 3, 4A, 4B and 5).
For the wedge transmission factors, the measured data for both low- and high-energy photon beams averaged for $90^{\circ}$ and $270^{\circ}$ collimator rotations were compared with the data received from the pilot hospitals. For the Varian and Siemens linear accelerators, wedge factors were measured for $15^{\circ}, 30^{\circ}$ and $45^{\circ}$ wedges. The Elekta linear accelerators are equipped with a motorised wedge, and only the $60^{\circ}$ angle was used for the audit measurements. The variation in wedge transmission factors was between $-2.2 \%$ and $4.8 \%$.

In this audit test, not all non-reference beam data requested from the hospitals were provided adequately.

This could be attributed to a lack of understanding on the objectives of the audit from the respective professionals because not all centres had been subjected to external audits previously. Also, some data were obtained entirely from the TPS because clinical tables, which are usually generated during commissioning, were not available. Some resident medical physicists did not have access to the original commissioning data. This indicates that there is poor recordkeeping, inadequate professional handover procedures as well as an overall lack of quality management.

\section{End-to-end dosimetry audits}

End-to-end dosimetry audit measurement results are presented for all three different reference points created during planning phase (Figure 7). Dose values were measured

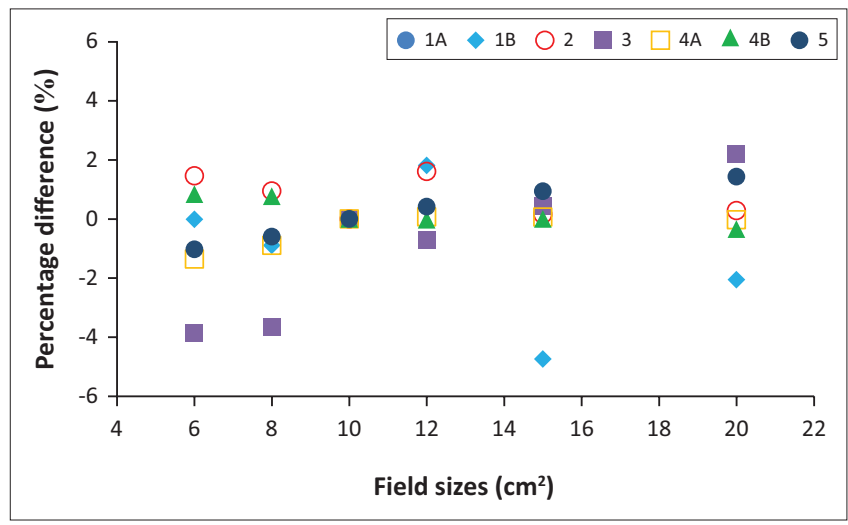

FIGURE 6: Comparison of measured and given output factors for five hospitals $(1 \mathrm{~A}, 1 \mathrm{~B}, 2,3,4 \mathrm{~A}, 4 \mathrm{~B}$ and 5$)$.

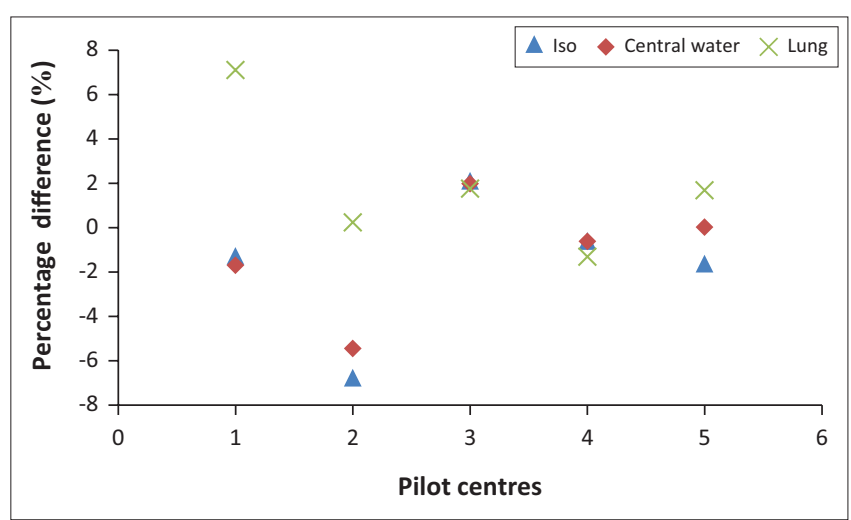

FIGURE 7: End-to-end results for five pilot hospitals (1, 2, 3, 4, and 5). 
at the reference points labelled Iso, Central water and Lung. The difference between the TPS dose value and linac measured values was plotted in Figure 7 with pilot hospitals indicated by number $1-5$.

The variation in the dose calculated by the TPS and the dose measured using the ionisation chamber in the CIRS IMRT phantom at the three reference points was within the range \pm $7.0 \%$, and the results outside $\pm 5.0 \%$, which is stated by the IROC group, require more interventions. It is recommended that a follow-up should be done to investigate the factors contributing to the high error margin difference in these measurements. Factors that could be considered may include chamber close to field edge, couch attenuation and the use of a $0.6 \mathrm{cc}$ ionisation chamber instead of smaller chambers as well as shortcomings with the dose calculation algorithms and setup errors.

\section{Conclusions}

The pilot study for the establishment of national radiotherapy and medical physics dosimetry end-to-end audit was successful. Up to $85 \%$ of audits comparison was within the $2 \%$ threshold, and $95 \%$ of audits comparison was within the $5 \%$ threshold. The results show that there is a need to conduct external dosimetry audits because some hospitals had radiation outputs outside the stated limits. A steering committee consisting of representatives like oncologists, radiation therapists, medical physicists and members of the regulatory body was formed to monitor the establishment of the programme. The IAEA and IROC recommend the 5\% tolerance for the dosimetry audits, and such measurement outside the tolerance requires thorough investigations.

Some of the challenges encountered during the pilot phase included lack of proper knowledge on the need for audits and inexperience staff or junior staff provided during audit measurements. Some of the initial data provided for comparisons, especially for non-reference audits, did not correspond to the request and could not be properly evaluated, as such follow-ups should be done. Some of the selected centres are far away from an audit laboratory, and proper funding for the logistics of developing national onsite audits is crucial. The success of these onsite pilot audits provided a platform to formulate and validate the prepared procedures required for rolling out a full national dosimetry audit programme. This pilot study was limited to reference beams and standard radiotherapy treatment. Further studies have been proposed to establish dosimetry audits for advanced radiotherapy techniques and small fields.

\section{Acknowledgements}

The authors would like to thank Fakazi Nhachisambe for his presence as a medical physics intern during data collection. The author wish to acknowledge the pilot hospitals for their contribution in making the staff and equipment available for the audit measurements.

\section{Competing interests}

The authors have declared that no competing interests exist.

\section{Authors' contributions}

H.V.M. was the main researcher in planning of dosimetry audits, onsite visits and measurement data collection. He was responsible for the preparation of the report, for presenting research results in both national and international events, and for the setup and preparation of writing and submission of the article. Z.N.M. contributed to the dosimetry pilot measurements planning, article input and corrections during the writing phase. N.M. contributed to the measurement data collection. D.v.d.M. contributed to the measurements guidance, approach and supervision on dosimetry audits and participated in analysis of all results.

\section{Funding}

The equipment used in this research were funded by the International Atomic Energy Agency (IAEA), and travelling was funded by the National Metrology Institute of South Africa (NMISA).

\section{Data availability statement}

Data sharing is not applicable to this article as no new data were created or analysed in this study.

\section{Disclaimer}

The views expressed in this article are the authors' own and not an official position of the institution or funder. Any other views from other sources have been referenced.

\section{References}

1. Khan FM. The physics of radiation therapy. 5th ed. Philadelphia, PA: Lippincott William and Wilkins; Wolters Kluwer Health; 2009.

2. Muhammad W, Ullah A, Mahmood K, Matiullah. Assessment of national dosimetry quality audits results for teletherapy machines from 1989 to 2015. J Appl Clin Med Phys. 2016;17(2):145-152. https://doi.org/10.1120/jacmp.v17i2.5984

3. Fraass B, Doppke K, Hunt M, et al. American Association of Physicists in Medicine Radiation Therapy Committee Task Group 53: Quality assurance for clinical radiotherapy treatment planning. Med Phys. 1998;25(10):1773-1829. https:// radiotherapy treatment planr
doi.org/10.1118/1.598373

4. Andreo P, Cramb J, Fraass BA, et al. IAEA Technical Report Series Number 430 Commissioning and quality assurance of computerized planning systems for radiation treatment of cancer. Vienna: International Atomic Energy Agency; 2004.

5. Starkschall G, Steadham RE, Wells $\mathrm{NH}$, et al. On the need for monitor unit calculations as part of a beam commissioning methodology for a radiation treatment planning system. J Appl Clini Med Phys. 2000;1(3):86-94. https://doi. org/10.1120/1.308251

6. ICRP. Preventing accidental exposures from new external beam radiation therapy technologies. ICRP Publication 112. Ann ICRP. 2009;39(4):1-2. https://doi. org/10.1016/j.icrp.2009.12.001

7. Podgorsak EB. Radiation oncology physics: A handbook for teachers and students. Vienna: International Atomic Energy Agency; 2005.

8. Attix $\mathrm{FH}$. Introduction to radiological physics and radiation dosimetry. Madison, Wisconsin: Wiley-VCH Verlag GmbH \& Co. KGaA; 1986. https://doi.org/ 10.1002/9783527617135

9. Bielajew AF. Fundamentals of radiation dosimetry and radiological physics. [homepage on the Internet].[cited n.d.]. Ann Arbor, Michigan; 2005. Available from: http://www-personal.umich.edu/ bielajew/DosimetryBook/book.pdf.

10. Podgorsak EB. Radiation physics for medical physicists. Berlin, Heidelberg: Springer-Verlag; 2006.

11. Izewska J, Svensson H, Ibbott G. Worldwide QA networks for radiotherapy dosimetry. Vienna: International Atomic Energy Agency, IAEA-CN-96-76; 2001. 
12. Girard F, Bouchard H, Lacroix F. Reference dosimetry using radiochromic film. J Appl Clin Med Phys. 2012;13(6):339-359. https://doi.org/10.1120/jacmp.v13i6.3994

13. Izewska J, Andreo P, Vatnitsky S, Shortt KR. The IAEA/WHO TLD postal dose quality audits for radiotherapy: A perspective of dosimetry practices at hospitals in developing countries. Radiother Oncol. 2003;69(1):91-97. https://doi.org/10.1016/ S0167-8140(03)00245-7

14. Izewska J, Bera P, Vatnitsky S. IAEA/WHO TLD postal dose audit service and high precision measurements for radiotherapy level dosimetry. Radiat Prot Dosim. 2002;101(1-4):387-392. https://doi.org/10.1093/oxfordjournals.rpd.a006008

15. Izewska J, Hultqvist M, Bera P. Analysis of uncertainties in the IAEA/WHO TLD postal dose audit system. Radiat Meas. 2008;43(2-6):959-963. https://doi. org/10.1016/j.radmeas.2008.01.011

16. Jornet N, Carrasco P, Beltrán M, et al. Multicentre validation of IMRT pretreatment verification: Comparison of in-house and external audit. Radiothe Oncol. 2013;112(3):381-388. https://doi.org/10.1016/j.radonc.2014.06.016

17. Eaton DJ, Bolton S, Thomas RA, Clark CH. Inter-departmental dosimetry audits: Development of methods and lessons learned. J Med Phys. 2015;40(4):183-189. https://doi.org/10.4103/0971-6203.170791

18. Budgell G, Berresford J, Trainer M, Bradshaw E, Sharpe P, Williams P. A national dosimetric audit of IMRT. Radiother Oncol. 2011;99(2):246-252. https://doi. org/10.1016/j.radonc.2011.03.016

19. Andreo P, Burns DT, Hohlfeld K, et al. IAEA TRS No.398. Absorbed dose determination in external beam radiotherapy. Vienna: International Atomic Energy Agency; 2000.

20. Anderson MD. Imaging and radiation oncology core [https://www.irochouston. mdanderson.org.].[cited n.d.] IROC Houston Quality Assurance Center, Texas; 2018. Available from: https://www.irochouston.mdanderson.org.
21. Rosenblatt E, Zubizarreta E, Izewska J, Binia S, Garcia-Yip F, Jimenez P. Quality audits of radiotherapy centres in Latin America: A pilot experience of the audits of radiotherapy centres in Latin America: A pilot experience of the
International Atomic Energy Agency. Radiat Oncol. 2015;10:169. https://doi.org/ International Atomic Energy Ag

22. Izewska J, Georg D, Bera P, et al. A methodology for TLD postal dosimetry audit of high-energy radiotherapy photon beams in non-reference conditions. Radiother Oncol. 2007;84(1):67-74. https://doi.org/10.1016/j.radonc.2007.06.006

23. Mizuno H, Sakata S, Yamashita W, et al. Application of a radiophotoluminescent glass dosimeter to nonreference condition dosimetry in the postal dose audit system. Med Phys. 2014;41(11):112104. https://doi.org/10.1118/1.4898596

24. Brunckhorst E, Gershkevitsh E, Ibbott G, et al. IAEA-TECDOC-1583. Commissioning of radiotherapy treatment planning systems: Testing for typical external beam treatment techniques. Vienna: International Atomic Energy Agency; 2008.

25. Almond PR, Cunningham JR, JG Holt, et al. AAPM TG 21. A protocol for determination of absorbed dose from high-energy photon and electron beams, Radiation Therapy Committee. Med Phys. 1986;10(6):741-771. https://doi.org/ 10.1118/1.595446

26. Almond PR, Biggs PJ, Coursey BM, et al. AAPM TG 51 protocol for clinical reference dosimetry of high-energy photon and electron beams. Med Phys. 1999;26(9): 1847-1870. https://doi.org/10.1118/1.598691

27. Cho SH, Lowenstein JR, Balter PA, Wells NH, Hanson WF. Comparison between TG-51 and TG-21: Calibration of photon and electron beams in water using cylindrical chambers. J Appl Clin Med Phys. 2000;1(3):108-115. https://doi.org/ 10.1120/1.308254

28. CIRS IMRT Thorax Phantom Manual, Model 002LFC. Tissue simulation and phantom technology [homepage on the Internet].[cited n.d.]. Norfolk, VA; 2018. Available from: https//www.cirsinc.com. 